\title{
Commentary on "Balancing the possibility of needing a future incontinence procedure versus a future urethral sling revision surgery-a tradeoff analysis for continent women undergoing pelvic organ prolapse surgery"
}

\author{
Abdelmageed Abdelrahman ${ }^{1}$ \\ Received: 2 April 2020 / Accepted: 7 April 2020 / Published online: 24 April 2020 \\ (C) The International Urogynecological Association 2020
}

This study [1] involved assessing nonpublic data from the California Office of Statewide Health Planning and Development (OSHPD) for the years 2005-2011. All women who underwent vaginal pelvic organ prolapse (POP) surgery during the study period were identified. As it was not possible to accurately identify a woman's preoperative continence status, the authors created two cohorts to compare the possible outcomes of POP surgery with or without a prophylactic sling procedure. The first cohort consisted of women who had a POP repair with a concurrent sling procedure (POP + sling), whereas the second cohort consisted of women who had undergone a POP repair without a concomitant stress urinary incontinence procedure (POP repair only). The primary outcome was a repeat surgical procedure for either a sling-related complication in the POP repair + sling group or a subsequent stress urinary incontinence procedure in the POP repair-only group. The authors defined surgery for a complication related to a sling procedure as any surgery with both a diagnosis and procedure related to sling revision.

A total of 81,314 women were included in the study cohort (38,456 underwent POP repair + sling and 42,858 underwent POP repair only). The POP repair + sling group were more likely to undergo future surgery for a sling complication compared with the POP repair-only group needing future stress urinary incontinence procedures. The authors concluded that a "wait and see approach" has the advantage of minimizing the potential risk of incontinence surgery-related complications by only treating symptomatic women motivated to have another procedure. This approach is also more cost effective.

The authors present an interesting study. Limitations of this study include the use of administrative data without preoperative examination information; therefore, the results may not be generalizable. It is also important to note that the endpoint of a surgical procedure omits women who are significantly bothered by their de novo stress urinary incontinence, but choose conservative measures.

\section{Compliance with ethical standards}

Conflicts of interest None.

\section{Reference}

1. Dallas K, Rogo-Gupta L, Syan R, Enemchukwu E, Elliott CS. Balancing the possibility of needing a future incontinence procedure versus a future urethral sling revision surgery: a tradeoff analysis for continent women undergoing pelvic organ prolapse surgery. Int Urogynecol J. 2020. https://doi.org/10.1007/s00192-020-04226-3

Publisher's note Springer Nature remains neutral with regard to jurisdictional claims in published maps and institutional affiliations.

Abdelmageed Abdelrahman

abdelmageed@hotmail.co.uk

1 Department of Urogynaecology, Liverpool Women's Hospital NHS Foundation Trust, Liverpool, UK 